\title{
Complex Systems Theory as a Shared Discourse Space for TESOL
}

DOI:

10.1002/tesj.317

Document Version

Accepted author manuscript

Link to publication record in Manchester Research Explorer

\section{Citation for published version (APA):}

Kostoulas, A., Stelma, J., Mercer, S., Cameron, L., \& Dawson, S. (2018). Complex Systems Theory as a Shared Discourse Space for TESOL. TESOL Journal, 9(2), 246-260. https://doi.org/10.1002/tesj.317

\section{Published in:}

TESOL Journal

\section{Citing this paper}

Please note that where the full-text provided on Manchester Research Explorer is the Author Accepted Manuscript or Proof version this may differ from the final Published version. If citing, it is advised that you check and use the publisher's definitive version.

\section{General rights}

Copyright and moral rights for the publications made accessible in the Research Explorer are retained by the authors and/or other copyright owners and it is a condition of accessing publications that users recognise and abide by the legal requirements associated with these rights.

\section{Takedown policy}

If you believe that this document breaches copyright please refer to the University of Manchester's Takedown Procedures [http://man.ac.uk/04Y6Bo] or contact uml.scholarlycommunications@manchester.ac.uk providing relevant details, so we can investigate your claim.

\section{OPEN ACCESS}




\title{
Complex Systems Theory as a Shared Discourse Space for TESOL
}

\author{
Authors' Accepted Manuscript \\ Achilleas Kostoulas, University Of Graz \\ Juup Stelma, University Of Manchester \\ Sarah Mercer, University Of Graz \\ Lynne Cameron, Open University and Freie Universität Berlin \\ Susan Dawson, University Of Manchester
}

\begin{abstract}
In this paper, we explore how insights from Complex Systems Theory might resonate with the experience of TESOL practitioners, and argue that complexity can function as a shared discourse space where connections might be drawn between research and practice. The paper has grown out of a meeting between TESOL practitioners and researchers at the [Name of University] Roundtable on Complexity Theory and English Language Teaching. It builds further on that discussion by exploring how language education practices and processes, that are familiar to us as education practitioners and researchers, can be understood in complexity-informed terms. To that end, we outline elements of Complex Systems Theory, which we believe resonate with what TESOL educators already know. These include a discussion of what complex systems are, how they operate, and how they evolve, all of which we illustrate with examples from our research and language education experiences. It is our hope that the complexity-informed perspective that we outline can provide teachers and researchers alike with an interpretive frame that may make more accessible the interconnected, sometimes unpredictable, invariably creative and intuitively recognisable nature of language education.
\end{abstract}

This paper is the product of a meeting between TESOL practitioners and researchers at the [Name of University] Roundtable on Complexity Theory and English Language Teaching, which took place in the [time and place of Roundtable], and which aimed to explore how Complex Systems Theory (CST) might be used to bridge the perceived gap between language learning research and teaching practice. In the spirit of the Roundtable, and following the lead of Author 3 (2014), this article looks to CST as an opportunity to further develop "a shared discourse space" for practitioners and researchers that may enhance "dialogue and cooperation for working together" (Author 3, forthcoming, p. 18). We believe this opportunity arises from the potential of CST to shape TESOL research and theory in ways that resonate more closely with the real world experience of English language teachers. To this end, the paper outlines our view of CST as a means to make the interconnected, sometimes unpredictable, and invariably creative nature of language education more accessible to shared reflection and investigation by researchers and practitioners. 
On one level, it is hoped that this paper can serve as an introduction to Complex Systems Theory and English language education, thereby making it accessible to those less familiar with the theory as yet. This is motivated by our belief that the phenomena which CST seeks to explain overlap considerably with our actual experiences as language educators. Throughout the paper, we explore this overlap by presenting key constructs from CST, and by drawing connections between this discussion and language teaching, with reference to both the ELT literature and our own experiential knowledge of ELT. Thus, we start with a discussion of the notion of 'system' in CST. We then talk about how complex systems function. We examine their propensity to remain in 'preferred states', and then we go on to discuss how CST can help to describe processes of change, such as the ones commonly encountered in language education. In doing so, we wish to make the case that the conceptual tools of CST are intuitively familiar to anyone engaged in the practice of language education.

Embedded in this introduction is a focus on the various affordances that we believe CST provides for practitioner reflection and researcher investigation. The discussion also contributes, directly and indirectly, to developing a shared vocabulary for what we perceive as the shared conceptual and discourse space for researchers and practitioners, which CST constitutes. After establishing this theoretical groundwork, we suggest that complexity-informed understandings of TESOL can be used to connect research-oriented and more practical work that is being done in the field.

\section{UNDERSTANDING COMPLEX SYSTEMS}

Complex Systems Theory is commonly associated with the study of systems. Systems, broadly construed, are defined as collections of "entities which interact in specific ways by virtue of their membership in the system" (Juarrero, 1999, p. 109). In education, for instance, a class could be viewed as a system because its members (teachers and learners) act and speak in certain ways when they are together. In addition to being associated with a space and a particular time, systems may have a historicity which influences their activity. So, for example, classroom behaviour will be a product of current dynamics as well as past experience that has been 'sedimented' in the system. Likewise, systems may be associated with possible future states (e.g., aspirations, goals, conceptualisations of ideal selves). It is perhaps important to note, at this point, that systems are conceptual frames, and that while the entities that make up a system might physically exist in the real world, the system as such only exists conceptually. Defining, or 'framing', a system is a conceptual move that we make in order to make sense of certain phenomena that we observe, and the system only comes into existence through this process of definition. Sometimes the boundaries of the system might suggest themselves by the observed world (e.g., schools and classes have welldefined physical and organisational limits), but it is common for these borders to be 'fuzzy'. In language education, for example, it may be analytically expedient to view a class as a system, taking into account its semi-permanent structure, the similarities in the learners' backgrounds and objectives, and the physical boundaries of the classroom; but when framing the system in such terms we must remain alert to the fact that its boundaries are permeated by rules and norms of the school culture, the influence of extracurricular events on the learners' affective states and more. In a sense, then, "the boundary of the system is neither purely a function of our description, nor is it purely a natural thing" (Cilliers, 2001, p. 141).

Complex systems are a special class of systems that are typically composed of multiple interconnected entities. What distinguishes them from other systems, however, is not the number of entities that a system comprises, but rather the dense web of relations that develop between them. This intricate web of interconnections means that the activity of every entity has direct or indirect repercussions on the entire system (Cilliers, 1998). In addition to the interconnections 
among system constituents, the dynamics of a complex system also rely on its interaction with other systems that are present in its environment (Prigogine \& Stengers, 1984). For instance, a class will interact with other 'proximate' systems such as curriculums, school as a whole, national cultures, other classes etc. The point is that, while we might choose to think of systems as discrete entities, their activity cannot be understood in isolation from other related systems with which they are interconnected. Sometimes, it can be useful to conceptualise these systems as being nested within each other (Davis \& Sumara, 2006). A language school, for instance, comprises multiple classes, each one of which may be viewed as a complex system in its own right; equally, it is nested in an education system which can be understood as a complex system as well. Helpful though such a hierarchical conceptualisation is, it may be more accurate to think of systems and their environment systems as a mesh of overlapping and interpenetrating systems with information flowing in all directions (Byrne \& Callaghan, 2014).

\section{STABILITY IN COMPLEX SYSTEMS}

Complex systems often seem to settle in fairly regular patterns of activity, or 'attractor states' (Other Author \& Author 3, 2008). There are a number of interactive phenomena that might reasonably be described as 'attractor states.' Classroom interaction, for example, often takes very recognisable shapes, such as the Initiation-Response-Feedback discourse pattern (Sinclair \& Coulthard, 1992), or learner-learner interaction with distinct modes of disputational, cumulative and exploratory talk (Mercer, 1995). In other words, although classroom interaction could evolve along any number of plausible trajectories, there seem to be some preferred patterns, which are analogous to what is described as attractor states in CST. Using a complexity perspective to describe a larger system, Author 1 (2014) has shown how a private language school in Greece appeared to remain entrenched in established pedagogical practices, including the transmissive teaching of decontextualized grammar, in the face of sustained top-down efforts to implement a communicative syllabus. In other words, among the range of possibilities that were potentially open to the system (the language school), transmissive teaching seemed to have the status of an attractor state from which the system seemed unable to escape.

What is important to note about attractor states is that they are not incidental features of a complex system. Rather, it is the structure and dynamics of the system that cause the activity to settle in these patterns, and to return to them even after minor perturbations. For instance, a teacher's and indeed also the learners' expectations, the structure of a textbook, the classroom layout and more, may 'act together' to exert a strong control on what learners are doing at any point in a lesson. Similarly, the 'cultural patterns of working' that the teacher and learners bring with them to a lesson may constrain the degrees of freedom that might otherwise be available to them. Moreover, many of us tend to have what sociologists have called a 'conservative impulse' (Marris, 1975), and hence teachers and learners alike may be averse to situations that lack the structuring comfort provided by experience, authority, history or culture (Prabhu, 1992). In the CST literature, the propensity of systems to stay within a 'preferred state' is often visually portrayed by means of a topographical metaphor: a plane is used to represent all the possible states in which a system can find itself (a 'state space'), and valleys and ridges in this place depict likelier or less likely states respectively (see Figure 1).

\section{[FIGURE 1 APPROXIMATELY HERE]}

The concept of attractors has been explicitly evoked in Author 4 (1999) to describe a state of 'minimal interaction' in a primary English language task in a Norwegian setting, which was viewed as a complex system. She suggests that the demands made on learners by cognitively challenging teacher questions resulted in very brief learner responses, which in turn led to more and more 
closed questions as the teacher attempted to elicit output. This 'attractor state' of minimal interaction is illustrated by point A in Figure 1. Only occasionally, when a learner subverted this pattern by taking initiative in the talk, combined with the teacher providing a personalised response, did the classroom activity move, if only briefly, into a state of more extended interaction (illustrated by point $B$ in Figure 1). Using this complexity-inspired analysis, Author 4 illustrates how language education activity may get trapped into a predictable pattern, and that shifting the activity to a different pattern requires a certain amount of effort. It further illustrates that a system is likely to return to its preferred state, unless there is some more fundamental and lasting change to the landscape of the state space.

When thinking about a complex system in an attractor state, whether this system represents a learner's mind, or a classroom, or the education provision of an entire country, it is important to remember that this situation is not static. An attractor might consist of several states through which a system traverses in a regular, or even an unpredictable (chaotic), sequence. Rather, even seemingly stable attractors exhibit dynamism, a condition described as dynamical stability, in which the system is in constant flux but this variability does not produce visible change. A variety of images have been used to metaphorically portray dynamical stability, including that of a river, which appears unchanging, even though water is constantly flowing downstream between the riverbanks. Similarly, systems may often appear to be stable, but this appearance of stability should not mask the fact that there is a constant flow of information and activity among the system constituents. Sometimes, small cumulative changes that result from such activity might lead to subtle reconfigurations in the dynamics of a system, thus creating the conditions for a dramatic change. Thinking of ELT as a very large system, the paradigm shift that ushered in the communicative approach in the late 1970s is an example of such a major and rapid restructuring, which happened after a relatively long period of dynamical stability, when behaviourist and structuralist approaches to teaching seemed unassailable.

\section{CHANGE IN COMPLEX SYSTEMS}

A distinctive feature of complex systems is that the intricate interconnections among their constituents can give rise to unexpected phenomena. These are often non-linear, meaning that the outcomes are not necessarily proportionate to the events that triggered them, and that it is often hard to make direct causal connections between triggering events and outcomes (Byrne \& Callaghan, 2014). One example of non-linearity, in language learning, might be when a teacher chooses to ignore a minor incident of disruptive behaviour in class, which might cascade into uncontrollable classroom dynamics, leading to problems later in time. More generally, as teachers we all know well, language learners' progress is seldom linear; learning often comes in spurts, interspersed by periods of apparent lack of progress or even regression (cf. Hohenberger \& PeltzerKarpf, 2009). In the CST literature, change is variously described as gradual, cumulative, sudden and/or unpredictable, which is consistent with what we know about the variety of change processes we observe in language education, including learning, motivation, teacher development, group dynamics, institutional change and curriculum reform. Appreciating the non-linear nature of change can be hugely empowering for language educators, because it helps to counter possible feelings of frustration caused occasionally by the mismatch between our efforts to affect change and actual outcomes.

One of the best known images of CST, which is associated with change, was put forward by the meteorologist Edward Lorentz in the early 1970s, to describe a surprising observation he made during his experiments with a dynamic mathematical model that simulated weather patterns. Apparently, even very small changes in the initial values he entered into the model (small fractions 
of a degree Centigrade), could produce substantial differences in the output of the simulation. In Complex Systems Theory, this property of systems is formally termed 'sensitive dependence on initial conditions', but it is more widely known as the 'butterfly effect', due to an evocative metaphor used by Lorentz in a conference paper delivered in 1972, where he suggested that disturbances in the atmosphere as minute as those produced by a butterfly flapping its wings might trigger a cascade of events ultimately causing unpredictable large-scale meteorological phenomena, such as a thunderstorm, thousands of miles away (Lorenz, 1972).

Sensitive dependence on initial conditions is relatively well-understood in language education, even though it has not always been interpreted through a complexity frame. One exception is Finch (2010), who has explored how "events that seem insignificant when they occur can grow to have major implications" (p. 424). Finch calls these events 'critical incidents', and in his research they included experiences of success, observing or talking to a friend or a family member, 'aha' moments and more. Finch suggests that these critical incidents were "the starting points (initial conditions) for significant changes in ... subsequent learning paths" (p. 430).

Language teachers and researchers seem intuitively aware of the importance of generating appropriate initial conditions. For example, a study conducted by Author 3 (2017) documented the belief among teachers that classroom dynamics are easiest to influence when learning groups are still being formed, and the importance of attending to classroom dynamics and motivation during the early stages of language courses is commonly stressed in the literature (e.g., Dörnyei, 2001; Dörnyei \& Murphey 2003). However, the challenge for researchers and teachers is both that the long-term outcomes of classroom activity are difficult to follow and that key incidents may appear too trivial to record as they take place. From our perspective as researching educators, the value of this and subsequent insights into change processes in language education is to provoke reflection on change, and to prompt awareness of the ways in which even seemingly trivial actions or exchanges could have larger effects.

Another hallmark of complex systems is that they produce emergent phenomena. These are systemwide phenomena, which are sometimes surprisingly intricate, but which come to existence without actual planning or central co-ordination (Davis \& Sumara, 2006). When thinking of individual learners, constructs like creativity or motivation are examples of emergent phenomena; at the class or school level, positive classroom dynamics, and the development of 'small cultures' (Holliday, 1999) would be examples. Emergent phenomena may be linked to attractor states; an emergent phenomenon may move into an attractor state, which, whilst still being dynamic (see previous section), could make the phenomenon appear more stable. Both the phenomena and the attractor states are simultaneously generated by the dynamics inside the system, and constrained (or channelled) by dynamics around the system. For example, in Authors 1 and 2 (2015), there is a description of how the dynamics inside a Norwegian EFL classroom gave rise to a 'performance intentionality', which refers to a keenness among young learners to engage in enthusiastic, theatrical behaviour during communicative tasks with the intention of entertaining their classmates. This intentionality emerged spontaneously and eventually 'took a life of its own', but, at the same time, it was constrained by influences such as curricular objectives, courseware content, and a sense of 'proper' school behaviour, which the teacher invoked when the theatrics became counterproductive. For us as language educators, emergence is a construct with great explanatory power, because it helps us to understand how highly organised activity, or attractor states, might come into existence in the absence of central organisation, or even despite efforts to control it.

In the discussion above, we looked into what complex systems are, and into some aspects of their activity, with a view to sharing our observation that many of the concepts and images used in complex systems theory have resonated with our lived experience as language educators. Given the 
recognisability of phenomena through this lens, in the following section, we want to argue that CST can function as a shared discourse space for researchers and practitioners, where it may be possible to transcend the research-practice divide.

\section{SHARING A DISCOURSE SPACE}

The discussion in this section is prompted by a problematisation, shared by the authors and other participants at the [Roundtable], about how research in ELT, and related domains of inquiry such as linguistics, psychology and education, is often framed in ways that are incompatible with the practicalities of everyday teaching. We acknowledge that there are instances when such a difference in perspective between research and practice is necessary, but we also believe there is a need to connect the two. Central to our concerns is the belief that any such attempts to bridge the researchpractice dichotomy cannot be imposed top-down, which is why we have deliberately avoided overspecifying the suggestions that follow. That said, we believe in the potential of a common discourse space, defined by a shared vocabulary and common ways of thinking, which can be used by practitioners and researchers in TESOL together, in our joint quest to develop new ways of understanding and improving our language learning and teaching experiences.

We believe that such a shared discourse space, which uses a complexity-inspired perspective as an interpretative frame for making sense of the classroom experience, would be particularly useful to teacher-led, and learner-led inquiry, such as Exploratory Practice (Hanks, 2015) or Action Research (Burns, 2010). Although such inquiry can be successfully implemented without recourse to CST, we maintain that using conceptualisations derived from CST offers a number of affordances on which researching educators and even learners can capitalise. To begin with, by viewing our professional context as a complex system, or rather a mesh of overlapping systems, we can become more sensitive to its relational nature. This, in turn, foregrounds the potential of even minor actions to have system-wide effects. Moreover, complexity-informed perspectives can be helpful in understanding order and change in our professional contexts. They can raise awareness of the sometimes opaque dynamics that underpin the more predictable elements of our professional experience, and can offer insights into the non-linear nature of change. Significantly, the insights generated by complexity-informed classroom research are likely to be readily accessible to practitioners, because they conceptually overlap with their experience more easily than is perhaps the case with decontextualized research. In addition, teachers' narratives of classroom experiences can be reported in ways with which research can readily engage.

We also believe that complexity-informed understandings of professional practice can function as a 'catalyst for reflection' (Widdowson, 2003, p. 27), which can drive professional development. Complex Systems Theory, in this sense, can function as a reflective framework, which can be especially helpful in making sense of our agency as educators. One way in which Complex Systems Theory can do this is by highlighting the subtle ways in which our professional action is shaped by the contextual dynamics of the systems of which we are integral elements. Secondly, such a perspective helps us to understand the non-linear ways in which our professional action relates to outcomes, and therefore provides theoretically grounded explanations for the mismatches between effort and outcomes. Finally, Complex Systems Theory provides insights into how our professional action impacts these systems of which we are part, and then recursively shapes our own behaviour. These insights are useful in raising our awareness of the effects our actions have on our professional contexts.

A shared discourse space, such as the one we are suggesting, can only develop through interaction. We believe that this interaction has already begun in the academic and professional literatures, where practitioners and researchers are increasingly turning to Complex Systems Theory to frame 
their insights (e.g., Kramsch, 2012; Maas, 2005; Author 3, 2013). Interaction is also beginning to take place at practitioner and academic conferences, where complexity-informed talks are becoming more common, and in journals and online environments, where complexity thinking is becoming increasingly visible. Interactions such as these, including the [Roundtable], have helped us, the authors of this paper, to move our own understanding of CST and its affordances for TESOL forward. However, we are concerned that many of the interactions have not always included both practitioners and researchers in the same space, nor have they always led to enhanced dialogue and cooperation. We would like this article to be one further step on a journey towards a shared discourse space enabled by CST. We hope that this journey will develop momentum, and look forward to other practitioners and researchers building on these tentative first steps.

\section{WORKS CITED}

Burns, A. (2010). Doing action research in English language teaching: A guide for practitioners. New York: Routledge.

Byrne, D., \& Callaghan, G. (2014). Complexity theory and the social sciences: The state of the art. New York: Routledge.

Cilliers, P. (1998). Complexity and postmodernism. London: Routledge.

Cilliers, P. (2001). Boundaries, hierarchies and networks in complex systems. International Journal of Information Management, 5(2), 135-147.

Davis, B., \& Sumara, D. J. (2006). Complexity and education: Inquiries into learning, teaching, and research. Mahwah, N.J.: Lawrence Erlbaum Associates.

Dörnyei, Z. (2001). Motivational strategies in the language classroom. Cambridge: Cambridge University Press.

Dörnyei, Z. \& Murphey, T. (2003). Group dynamics in the language classroom. Cambridge: Cambridge University Press.

Finch, A. (2010). Critical incidents and language learning: Sensitivity to initial conditions. System, 38(3), 422-431. doi: 10.1016/j.system.2010.05.004

Hanks, J. (2015). Language teachers making sense of EP. Language Teaching Research 19(5), 612633. doi: $10.1177 / 1362168814567805$

Hohenberger, A., \& Peltzer-Karpf, A. (2009). Language learning from the perspective of nonlinear dynamic systems. Linguistics, 47(2), 481-511. doi: 10.1515/LING.2009.017

Holliday, A. (1999). Small cultures. Applied linguistics, 20(2), 237-264.

Juarrero, A. (1999). Dynamics in action: Intentional behavior as a complex system. Cambridge, Mass.: MIT Press.

Kramsch, C. (2012). Why is everyone so excited about complexity theory in applied linguistics? Mélanges CRAPEL, 33, 9-24.

Lorenz, E. (1972). Does the flap of a butterfly's wings in Brazil set off a tornado in Texas? Presented at the 139th annual meeting of the American Association for the Advancement of Science.

Maas, J. (2005). Principled practice: New science for the classroom. In T. Hatch (Ed.) Going public with our teaching: An anthology of practice. New York: Teachers College Press.

Marris, P. (1975). The conservative impulse. The Urban Review, 8(4), 288-299. doi: 10.1007/BF02178898

Mercer, N. (1995). The guided construction of knowledge: Talk amongst teachers and learners. Multilingual Matters.

Prabhu, N. S. (1992). The dynamics of a language lesson. TESOL Quarterly, 26(2), 225-241. doi: $10.2307 / 3587004$

Prigogine, I., \& Stengers, I. (1984). Order out of chaos: Man's new dialogue with nature. Boulder, CO: New Science Library: Distributed by Random House.

Sinclair, J., \& Coulthard, M. (1992). Towards an analysis of discourse. In Coulthard, M. (Ed.) Advances in spoken discourse analysis (pp. 1-34). London: Routledge. 
Widdowson, H. G. (2003). Expert beyond experience: Notes on the appropriate use of theory in practice. In D. Newby (Ed.) Mediating between theory and practice in the context of different learning cultures and languages (pp. 1-6). Graz: European Centre for Modern Languages, Council of Europe. 